\title{
Species Index
}

Page numbers in italics refer to the subject of the papers

Aedes albopictus, 281-287

Agaricus, 374 campestris, 372, 373

Allium, 17 cepa, 185-195

kachrooi, 22 schoenoprasum, 172 subvillosum, 185-195

Amphicarpaea bracteata, 347, 352

Arianta arbustorum, 115

Armadillidium, 81

aquaticus, 85

nasatum, 425

vulgare, $419,423,424$

Artemia salina, 342

Avena spp, 352

agadiriana, 403

barbata, 403, 409

canariensis, 403-411

fatua, 409

Bidens cynapiifolia, 142

discoidea, 142

frondosa, 142

pilosa, 139-143

sandvicensis, 140

tripartita, 142

Biomphalaria glabrata, 174

Biston betularia, 429

Bombyx mori, 18

Brassica spp, 392

Bufo boreas, 413

Bulinus cernicus, 174

Cactoblastis cactorum, 360

Callimorpha quadripunctaria, 87-92

Ceriagrion, 341

tenellum, 342

Cestrum, 268

Chorthippus brunneus, 20, 22

jucundus, 17-23

parallelus, 17-23

vagans, 17-23

Chortoicetes terminifera, 20, 22

Chrysochraon dispar, 17, 20

Circium undulatum, 247

Clarkia biloba, 410

lingulata, 410

xantiana, 105

Clausilia itala, 115

Climacium americanum, 223-238

var. kindbergii, 235

dendroides, 237

Cochlodina laminata, 113-120

Coenagrion puella, 345

Columba livia, 413

Crepis, 22 capillaris, 377-385

Cucumis sativa, 372,374

Cygnus columbianus, $73-80$

cygnus, 73-80

olor, $73-80$
Dendrocoelum lacteum, 17

Diataraxia oleracea, 329

Dichroplus pratensis, $145-159$

Drosophila, 63, 65, 100, 124, 151, 157, 158, 177, 215, 221, 230, $263-269,328,342,355,359,362,364,372,374,395-401$, 427

albomicans, 269

albostrigata, 269

americana, 256, 259

busckii, 371-375

buzzatii, 355-365

cardinoides, 263

flavipilosa, 263-269

guaramunu, 263

kohkoa, 269

littoralis, 255

lummei, 255-262

mediopunctata, 263-269

melanogaster, 18, 63, 67, 192, 193, 215-222, 223-231, 255, $260,261,319,363,372,395-401$

mojavensis, 372,374

nasuta, 263, 269

nebulosa, 263-269

novamexicana, 256, 259

polymorpha, 263

pseudoobscura, 267

simulans, 255, 261

subobscura, 55-66

suboccidentalis, 372, 374

sulfurigaster, 269

texana, 259, 260

virilis, 255-262

willistoni, 263-269, 410

Eichhornia paniculata, 271-280

Enallagma, 341

Enteromorpha sp., 420

Erysiphe fischeri, 347

graminis sp. tritici, 247

Euchorthippus chopardi, 17-23

pulvinatus, 17-23

Eyprepocnemis plorans, 20, 22

Flavobacterium spp., 414

Fritillaria, 17

Gammans duebeni, 419, 424

Gammarus oceanicus, 85

Gibasis linearis, 384

Gilia achilleifolia, 106

Glycine argyrea, 347-353

canescens, 347, 348, 351, 352

$\max , 247$

Haplopappus aureus, 43

venetus, 43

Helianthus annuus, 43

Heteracris littoralis, 20

Hordeum vulgare, 305-313

ssp. spontaneum, 239-250

ssp. vulgare, 241, 243

Hypochoeris, 22, 384 
Ipomoea hederacea, 106 purpurea, 105

Ischnura damula, 341, 343, 345

demorsa, 341, 343, 345

elegans, 342, 345

graellsii, 341-346

pumilo, 342

ramburi, 344, 345

Isotoma anaethifolia, 290

axillaris, 290

petraea, 289-295

Lacerta vivipara, 131-138

Lamium album, 87

Layia discoidea, 29-44

glandulosa, 29-44

ssp. glandulosa, 29-44

lutea, 29-44

Leptysma argentina, 154

Lestes barbarus, 342

dryas, 342

virens, 342

viridis, 342

Lilium, 17

Lolium multiflorum, 45, 311

perenne, 45-53, 221, 311

temulentum, 45-53

Lotus corniculatus, 387

Lupinus texensis, 248

Lymnaea peregra, 169-175

stagnalis, 174

Melanoplus femur-rubrum, 21

Mimulus cupriphilus, 111

guttatus, 111

Monodelphis domestica, 251, 253

Musca domestica, 315-321

Muscari comosum, 185-195

Myrmeleotettix maculatus, 20, 203, 379, 384

Najas marina, 384

Nezara viridula, 161-167

Nicotiana rustica, 105

Oedaleonotus enigma, 154

Omocestus panteli, 17-23

Oncorhynchus mykiss, 413-417

Opuntia, 355

Orchestia gammarella, 419

Panaxia dominula, 91

Papaver bracteatum, 367-370

orientale, 367-370

somniferum, 367,368

Parapleurus alliaceus, 17, 21

Passer domesticus, 76

Pellia borealis, 237

epiphylla, 237

Pentaglottis sempervirens, 87

Phakopsora pachyrhizi, 347-353

Phaseolus 430-431

acutifolius, 430

coccineus, 430

lunatus, 430, 431

vulgaris, 430
Phaulacridium, 158

Plagiochila asplenioides, 237

Platorchestia platensis, 85

Polytrichum juniperinum, 331-340

Porcellio, 81

dilatatus, 85

Porcellionides virgatus, 90

Primula vulgaris, 121-124

Pseudococcus affinis, 203

Puccinia coronata, 352

Puschkinia libanotica, 384

Pyrrhosoma, 341

Ramaria, 372, 374

Rumina decollata, 174

Salmo trutta, 297-304

ssp. dentax, 297

macedonicus, 297

macrostigma, 297

mediterranea, 302

pelagonicus, 297, 298

peristericus, 297

Sarracenia purpurea, 177-183

Schistocerca, 384

Scilla autumnalis, 185-195

sibirica, 193

Secale cereale, 125-130, 197-204

montanum, 125

Senecio squalidus, 43, 88, 139, 143, 393

vulgaris, $43,139,142,143,347,391-393$

Sitophilus oryzae, 323-330

Sminthopsis crassicaudata, 17, 251, 253

Solanum goniocalyx, 429

tuberosum, 429

Solonopsis invicta, 93-103

richteri, 93-103

Sorghum vulgare, 247

Sphaeroma rugicauda, 81-85, 419-425

Sphagnum, 237

Stethophyma grossum, 17, 21

Symphytum officinale, 87

Synchytrium decipiens, 347, 352

Taraxacum officinale, 247

Tettigidea lateralis, 384

Tragopogon dubius, 247

Tribolium, 223, 328

Trichopoda pennipes, 166

Trichosurus vulpecula, 251-254

Trifolium pratense, 311

repens, 387-390

Triticum aestivum, 247

Triturus cristatus, 17, 22

helveticus, 17,22

Tulbaghia, 17, 22

Turnera ulmifolia, 106

var. angustifolia, 105-112

Urtica dioca, 88

Vicia faba, 185

Zea mays, 384

Zootoca vivipara, see Lacerta vivipara 